\title{
L'OBBLIGO DI PATIENTIAM PRAESTARE NEL CONTESTO DI D. 39.3.2 (PAUL I.49 AD ED.). IL CASO DELLA FOSSA AGRORUM SICCANDORUM CAUSA DI CUI AI \$ 1 E 2
}

\author{
THE OBLIGATION OF PATIENTIAM PRAESTARE WITHIN \\ D. 39.3.2 (PAUL I.49 AD ED.). THE CASE OF FOSSA AGRORUM \\ SICCANDORUM CAUSA AS REFERRED IN \$\$ 1 AND 2
}

\author{
SARA PILLONI \\ sarapilloni@virgilio.it \\ UNIVERSITÀ DEGLI STUDI DI TRIESTE ${ }^{1}$
}

[RECIBIDO: 31/12/2018; ACEPTADO: 06/05/2019

http://dx.doi.org/10.25267/Riparia.2019.v5.06

\section{RIASSUNTO}

Il presente contributo propone un'analisi esegetica del testo di cui a D. 39.3.2.1 e 2 (Paul. 49 ad ed.) nel più ampio contesto del testo paolino di cui a D. 39.3.2 (Paul. 49 ad ed.). L'esegesi si concentra in particolare sull'obbligo di patientiam praestare come titolo di responsabilità alternativo a quello principale di restitutio suis sumptibus esperibile attraverso l'actio aqaue pluviae arcendae (di seguito a. a. p. a.). Nei paragrafi indicati, letti alla luce dei corrispondenti testi dei Basilici, è possibile individuare nell'esercizio dell'a. a. p. a. a titolo di patientiam praestare, oltre ad una forma di tutela dello stato delle proprietà fondiaria di entrambi i proprietari confinanti, anche una forma di regolamentazione dell'uso e della produttività dei fondi stessi, a vantaggio della miglior gestione delle risorse rurali.

\footnotetext{
${ }^{1}$ Dottore di ricerca in Diritto Romano e Cultore della materia, Cattedra di Istituzioni di Diritto Romano, Università degli studi di Trieste, Dipartimento di Scienze Giuridiche, del Linguaggio, dell'Interpretazione e della Traduzione, Sezione di Studi Giuridici, Piazzale Europa, 1 - 34127 - Trieste.

S. Pilloni, "L'obbligo di patientiam praestare nel contesto di D. 39.3.2 (Paul. I 49 ad ed.). Il caso della fossa agrorum siccandorum causa di cui ai \$\$ 1 e 2", RIPARIA 5 (2019), 139-165.
} 
PAROle-Chiavi: actio aquae pluviae arcendae, acque, scolo, proprietà fondiarie, ambiente

\begin{abstract}
This paper contains an analysis of D. 39.3.2.1 and 2 (Paul. 49 ad ed.) within the general framework of D. 39.3 .2 (Paul. 49 ad ed.). The exegesis focuses on the obligation of patientiam praestare as an alternative to the main one of restitutio suis sumptibus, both of them typical features of the actio aqaue pluviae arcendae (hereafter a.a.p.a.). Through the comparison between the texts above mentioned and the corresponding ones from the Basilika, the a.a.p.a. and the obligation of patientiam praestare outline a type of regulation in the use and productivity of the adjoining lands, for the better management of rural resources, beyond the traditional use as a preventive remedy to protect particular interests of land owners.
\end{abstract}

KEY WORDS: actio aquae pluviae arcendae, water, drainage, lands, environment 


\section{Introduzione}

Il celebre testo di Paolo di cui a D. 39.3.2 inquadra una serie di casi specifici nei quali la concessione dell'actio aquae pluviae arcendae (di seguito a. a. p. a.) appariva controversa rispetto alle condizioni generali della sua applicabilità ed ai principi regolatori del rapporto di servizio che legavano i due fondi coinvolti, vale a dire quello superiore e quello inferiore, secondo quanto espresso nel principium del testo paolino:

\section{39.3.2 pr (Paul. 49 ad ed.): In summa tria sunt, per quae inferior locus superiori servit, lex, natura loci, vetustas: quae semper pro lege habetur, minuendarum scilicet litium causa.}

Le fattispecie che vengono in rilievo nei paragrafi successivi, in rapporto alla regola generale, esemplificano manifestazioni peculiari della relazione tra ciascuno di tali principi regolatori e la legittimazione all'esercizio dell'a. a. p. $a$.

Nell'ambito di tale inquadramento, assume un certo interesse ai fini di una più articolata indagine sui rapporti reciproci tra fondi confinanti e, più in generale, sulla gestione del territorio rurale nell'esperienza antica, la questione dell'individuazione di un obbligo di patientiam praestare come titolo di responsabilità alternativo cui il proprietario-convenuto poteva essere chiamato a rispondere rispetto a quello principale di restitutio suis sumptibus, ovverosia l'obbligo di rimessione in pristino dello stato dei $\operatorname{luoghi}{ }^{2}$.

\footnotetext{
2 Dell'a. a. p. a. nel suo rapporto con la gestione delle risorse idriche nel mondo antico e la tutela dell'ambiente e del paesaggio, si è ampiamente occupata la dottrina romanistica. Con particolare riferimento a quest'ultima tipologia di contributi in tema di a. a. p. a. pare doveroso citare A. Di PORTO, "La tutela della "salubritas" fra editto e giurisprudenza. Il ruolo di Labeone. I - Acque", BIDR 91, 1988, 459 ss.; ID., "La tutela della "salubritas" fra editto e giurisprudenza. Il ruolo di Labeone. II. Cloache e salubrità dell'aria”, BIDR 92, 1989, 271 ss.; ID., La tutela della "salubritas" fra editto e giurisprudenza: Il ruolo di Labeone, Milano, 1990 (le citazioni nel testo si riferiscono alla monografia); F. MusuMECI, "Tutela dell'ambiente e diritto romano", Index 20, 1992, 564 ss.; G. FrANCIOSI, "Regime delle acque e del paesaggio in età
} 
In termini generali, l'obbligo di patientiam praestare consisteva nell'imposizione al proprietario o possessore di un bene del dovere di sopportare il compimento di una determinata attività sul proprio bene da parte di un soggetto estraneo ${ }^{3}$ : della classicità di tale obbligo si è ampiamente discusso in dottrina, anche con riferimento ad una serie di testi in tema di $a$. $a . p$. $a$. che la configurano quale contenuto alternativo e separato rispetto all'obbligo di ripristino dello stato dei luoghi ${ }^{4}$.

repubblicana", in Uomo, acqua e paesaggio. Atti dell'incontro di studio sul tema "Irregimentazione delle acque e trasformazione del paesaggio antico". S. Maria Capua Vetere - 22-23 novembre 1996, Roma, 1997, 11 ss.; M. FIorentini, "Precedenti di diritto ambientale a Roma? I. la contaminazione delle acque", Index 34, 2006, 353 ss.; ID., "Equilibri e variazioni ambientali nella prospettiva della tutela processuale romana", in E. HERMON (dir.), Société et climats dans l'Empire romain. Pour une perspective bistorique et systémique de la gestion des ressources en eau dans l'Empire romain, Préface de L. LabrunA, Napoli, 2009, 69 ss.; W. WolodkiewicZ, "Protection de l'environnement naturel et gestion de l'eau d'hier et d'aujourd'hui', in R. BEDON, E. HERMON (éds.), Concepts, pratiques et enjeux environnementaux dans l'Empire romain, Limoges, 2005, 53 ss. Una particolare menzione meritano i recenti contributi in tema di a. a. p. a. editi in Jus. Rivista di Scienze Giuridiche, a cura della Facoltà di Giurisprudenza dell'Università Cattolica di Milano: il n. 2 (2014) della rivista ospita infatti una serie di interessanti approfondimenti svolti in occasione dell'incontro di studi Terra, acqua, diritto. Giovani romanisti milanesi incontrano Gérard Chonquer, tra i quali si ricordano per il tema qui d'interesse, oltre a L. MAGANZANI, "Introduzione a 'Terra, acqua, diritto'. Giovani romanisti milanesi incontrano Gérard Chouquer", 207 ss., anche N. DONADIO, "La tutela dal rischio di danni da aqua pluvia nelle soluzioni giurisprudenziali tra tarda repubblica ed età severiana: a proposito di D. 39.3.2.5 (Paul. 49 ad ed.)", 231 ss.; F. PulitanÒ, "D. 39.3.4: Casistica giurisprudenziale in tema di actio aquae pluviae arcendae tra prerogative dei privati e interessi pubblici”, 255 ss.; F. SCOTTI, "Actio aquae pluviae arcendae e fossae agrorum siccandorum causa factae: per un'esegesi di D. 39.3.2.1,2,4,7 alla luce delle tecniche agronomiche antiche", 273 ss. Di quest'ultima studiosa si ricorda inoltre F. SCOTTI, "Diritto e agronomi latini: un caso in tema di actio aquae pluviae arcendae", Agri Centuriati 10, 2013, 9 ss.

3 Secondo la definizione che si può leggere in A. BERGER, v. Patientiam praestare, in Encyclopedic Dictionary of Roman Law, Philadelphia 1953 (rist. 1991), 620 e ripresa da L. PARENTI, "Osservazioni sul "patientiam praestare" in Labeone", in Teoria e storia del diritto privato (bttp://www.teoriaestoriadeldirittoprivato.com), V (2012), 1. A. BERGER collega tale obbligo all'ipotesi in cui un soggetto diverso dal proprietario del fondo (colono, schiavo o il precedente proprietario) abbia costruito sullo stesso un opus poi rivelatosi minaccioso per il fondo vicino: nel caso in cui non fosse il proprietario del fondo a provvedere alla distruzione dello stesso, avrebbe potuto farlo il vicino a sue spese, poi rimborsabili, dovendo il primo tollerare che quest'ultimo entrasse sul suo fondo per il compimento delle necessarie attività.

4 Di tali aspetti si sono occupati in modo specifico L. PARENTI, "Osservazioni sul "patientiam praestare"...", 3 ss. e già F. PETERS, "Das "patientiam praestare" im klassischen

"L’obbligo di patientiam praestare nel contesto di D. 39.3.2 ..." 
Tale obbligo compare in numerosi testi del titolo 3 del libro 39; vi è però da sottolineare una sostanziale differenza tra tali fattispecie e quelle di cui al complesso del frammento n. 2.

Le prime infatti attengono ad ipotesi nelle quali l'obbligo di patientiam praestare viene riconosciuto in capo a soggetti rispetto ai quali l'a. a. p. a. non potrebbe essere direttamente esercitata per la sola restitutio suis sumptibus per ragioni diverse: si tratta, infatti, di casi nei quali la questione rileva in termini di contrapposizione tra dominus auctor e dominus non auctor e, conseguentemente, di legittimazione passiva all'azione.

Più dettagliatamente, l'obbligo di patientiam praestare emerge nei casi discussi in D. 39.3.4.2 (Ulp. 53 dig.) e D. 39.3.5 (Paul. 49 ad ed.), per l'ipotesi di opus (sepulchri aedificatio nel primo caso) realizzato dal colono ignorante ovvero insciente domino; D. 39.3.11.2 (Paul. 49 ad ed.), per il caso di pluralità di domini tra i quali uno fosse non auctor, al quale si ritiene qui di aggiungere anche quello trattato in D. 39.3.11.6 (Paul. 49 ad ed.) per un problema di glarea iniecta aut fossa limo repleta, in quanto il complesso del testo di Paolo attiene ad un'ipotesi di comproprietà del fondo, circostanza questa che farebbe propendere per un inquadramento del caso ivi contemplato nei termini di una contrapposizione tra dominus auctor e dominus non auctor, e infine D. 39.3 .12 (Paul. 16 ad Sab.) per il caso

römischen Nachbarrecht”, SDHI 35, 1969, 136 ss. in relazione all'obbligo di patientiam praestare con riferimento tanto all'a. a. p. a. quanto all'interdictum quod vi aut clam. Tale obbligo resta ovviamente al di fuori dell'analisi condotta da R. CARDILLI, L'obbligazione di 〈praestare» e la responsabilità contrattuale in diritto romano (II sec. a.C. - II sec. d.C.), Milano, 1995 , in quanto il patientiam praestare in questo caso non rappresenta il contenuto di un'obbligazione assunta dalle parti, alla cui violazione consegue la responsabilità contrattuale del debitore, ma costituisce piuttosto il contenuto di un obbligo diverso collegato al rapporto di vicinanza che lega i proprietari dei due fondi. CARDILLI, però, menziona D. 39.3.2.6 (Paul. 49 ad ed.) a p. 242 - 243 nota 28 nell'ambito di un discorso più generale relativo all'obbligo di praestare incombente sul colono e al damnum causato dal vitium loci, leggendo peraltro l'opinione espressa da Namusa nel senso di considerare il dominus del fondo inferiore "tenuto a risarcire il danno indipendentemente dall'esistenza dell'opera dell'uomo, salvo che per l'opera agri colendi causa facta". 
del dominus nuovo acquirente del fondo obbligato.

A tal proposito, una rapida disamina dei corrispondenti testi nei Basilici, ove pervenuti, ci mostra del resto come in essi ci si

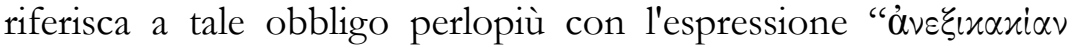
$\chi \varrho \varepsilon \omega \sigma \tau \circ \tilde{\sigma} \iota \nu "$, differentemente dai casi di cui ai D. 39.3.2 (Paul. 49 ad ed.), ove le espressioni utilizzate mutano a seconda della fattispecie considerata, adattandosi al dovere di cui il proprietario del fondo obbligato doveva di volta in volta rispondere.

Nell'ambito di tale testo, infatti, l'obbligo in questione pare assumere contorni in parte differenti da quelli segnalati espressamente con patientiam praestare, che resta piuttosto strettamente collegata alle peculiari vicende che attengono la proprietà del fondo, per assumere i contorni di una sorta di tutela minima garantita nel caso di malfunzionamento di un opus utile al fondo causato dall'acqua piovana.

Si tratta di un aspetto che emerge con particolare riferimento ai $\iint 1$ e 2 del testo paolino, in relazione a due peculiari ipotesi di aquae restagnatio causate dal mancato mancato spurgo da parte del proprietario del fondo di una fossa adibita al prosciugamento dei campi e collocata sulla sua proprietà.

Attraverso l'analisi di tali fattispecie e di alcune questioni relative alla ricostruzione delle soluzioni giuridiche ivi adottate, anche con l'ausilio del corrispondente testo dei Basilici, è possibile individuare nell'esercizio dell' $a$. $a . p$. . $a$., anche a titolo di patientiam praestare, una forma di tutela dello stato delle proprietà fondiaria, in favore di entrambi i proprietari dei fondi confinanti, ma che contribuisse altresì ad una regolamentazione dell'uso e della produttività dei fondi stessi, a vantaggio dunque della miglior gestione delle risorse rurali. 


\section{L'ipotesi di aquae restagnatio di cui a D. 39.3.2.1 (Paul. 49 ad ed.): il limite del pati in capo al dominus del fondo confinante.}

La prima ipotesi oggetto di analisi è quella di cui a $\mathrm{D}$. 39.3.2.1 (Paul. 49 ad ed.), relativo appunto ad un peculiare caso di aquae restagnatio:

D. 39.3.2.1 (Paul. 49 ad ed.): Apud Labeonem proponitur fossa vetus esse agrorum siccandorum causa nec memoriam extare, quando facta est: hanc inferior vicinus non purgabat: sic fiebat, ut ex restagnatione eius aqua fundo nostro noceret. Dicit igitur Labeo aquae pluviae arcendae cum inferiore agi posse, ut aut ipse purgaret aut te pateretur in pristinum statum eam redigere.
Bas. 58. 13. 2. 1, VIIA p. 2678 Scheltema: O





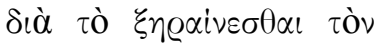

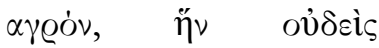



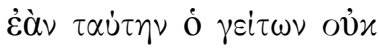



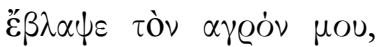



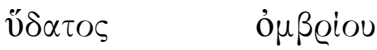

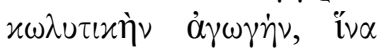

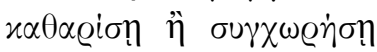
$\mu$ oı $x \alpha \theta \alpha \varrho i \sigma \alpha \iota$.

Sch. 1 a Bas. 58. 13. 2, VIIIB Scheltema, p.


$\dot{\varepsilon} \nu \tau \alpha \tilde{v} \theta \alpha \quad \mu \grave{\eta} \quad \tau$ òv $\theta \dot{\varepsilon} \alpha$ von்

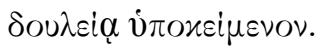

Il caso riportato da Paolo ed esaminato da Labeone riguarda un'ipotesi di ristagno delle acque causato dal mancato spurgo da parte del proprietario del fondo inferiore di una vecchia fossa adibita al prosciugamento dei campi e collocata, si ritiene, proprio sul suo fondo ${ }^{5}$ e del cui originario scavo non si ha

${ }^{5}$ La presenza della fossa pare sia da intendersi sul fondo inferiore, anche alla luce del 
memoria.

Secondo Labeone, il proprietario del fondo superiore, a vantaggio del quale opera la fossa stessa e che si assume messo in pericolo dall'incuria del proprietario del fondo inferiore, è legittimato ad esercitare contro quest'ultimo l'a. a. p. a., chiedendo che egli provveda allo spurgo della fossa (ipse purgaret = raAacion) oppure che sia tenuto a sopportare che il proprietario del fondo superiore ripristini personalmente il corretto funzionamento della

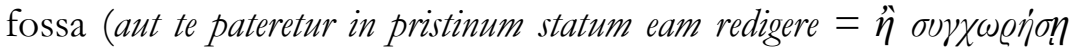



L'opus in questione era dunque utile alla coltivazione del fondo, la cui risalenza nel tempo era tale da aver fatto perdere la memoria stessa della sua costituzione ${ }^{6}$; si trattava inoltre di un opus agri colendi causa, come viene espressamente qualificata la fossa in questione in D. 39.3.1.4 (Ulp. 53 ad ed.) e dunque l'a. a. p. a. non poteva essere concessa per rimuovere l'opus ${ }^{7}$.

L'azione veniva dunque data per la riparazione dell'opus stesso nel caso in cui il cattivo stato di conservazione, con il concorso dell'acqua, risultasse potenzialmente dannoso per il

corrispondente testo dei Basilici, o almeno pare che così debba tradursi $\mathrm{O}$ vं $\pi 0 x \varepsilon i \mu \varepsilon v o \varsigma$

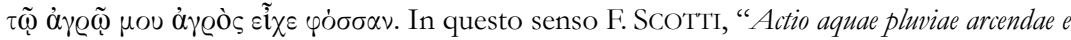
fossae agrorum siccandorum...", 276 e nota 13 e 305, in adesione a quanto già rilevato da P. BONFANTE, Corso di diritto romano. La proprietà, vol. II, Milano 1966 (ristampa corretta della I edizione a cura di Giuliano Bonfante e di Giuliano Crifò con l'aggiunta degli indici delle fonti), 498 ed ipotizzato da M. SARGENTI, Actio aquae pluviae arcendae. Contributo alla dottrina della responsabilità per danno nel diritto romano, Milano 1940, 56.

${ }^{6} \mathrm{Si}$ segue qui l'esegesi del testo proposta da ultimo da F. SCOTTI, "Actio aquae pluviae arcendae e fossae...", 276 ss.

7 Si rileva qui brevemente che nuovi spunti esegetici sono proposti in relazione al testo di cui a D. 39. 3. 1.4 (Ulp. 53 ad ed.) da F. SCOTTI, "Brevi riflessioni su Dig. 39.3.1.4,5,9 Ulp. 53 ad ed.", RIPARIA 1, 2015, 133 ss. e Id., "Actio aquae pluviae arcendae e «piccola bonifica agraria»: un esempio dalle fonti giustinianee", in Mater Familias. Scritti per Maria Zabtocka, Warszawa, 2016, 725 ss. = JusOnline 3, 2016, 32 ss. (le citazioni nel testo si riferiscono al contributo dell'Autrice pubblicato in JusOnline).

"L’obbligo di patientiam praestare nel contesto di D. 39.3.2 ..." 
proprietario del fondo confinante ${ }^{8}$.

La fossa in questione trovava fondamento nella vetustas e tale circostanza, conformemente a quanto statuito nel principium del testo paolino, avrebbe legittimato l'esercizio dell'a. a. p. a.: essendo stato causato il mutamento dello stato del fondo dal malfunzionamento di un opus legittimato dalla sua risalente presenza sul fondo, e non dalla sua distruzione, l'azione in questione avrebbe potuto essere esercitata anche per la manutenzione dello stesso?

Labeone, nel concedere l'azione, distinguerebbe poi ulteriormente sul piano della tutela prevista in favore del proprietario del fondo superiore: da un lato, infatti, egli avrebbe il diritto di agire per chiedere al proprietario del fondo inferiore la manutenzione della fossa ovvero, dall'altro, di provvedere personalmente allo spurgo, essendo tenuto il proprietario del fondo inferiore a sopportare che se ne occupi lui.

La previsione, però, non è considerata unanimamente originaria; ad essere stata sospettata di intervento compilatorio è in particolar modo l'ultima parte del testo, contenente il riferimento al dovere di patientiam praestare incombente sul proprietario del fondo inferiore ed azionabile per mezzo dell' $a$. $a$. p. $a^{10}$. La dottrina più recente, tuttavia, tende ad escluderlo ${ }^{11}$.

8 In questo senso si veda già F. SITZIA, Ricerche in tema di "actio aquae pluviae arcendae". Dalle XII tavole all'epoca classica, Milano, 1977, 87.

9 Sul punto nuovamente F. SiTZIA, Ricerche in tema..., 82 ss. e Id., "Aqua pluvia e natura agri”, Iura, 50, 1999, 63 e 68.

10 Secondo P. Bonfante, Corso di diritto romano..., II, 499-500 e G. BranCA, "La responsabilità per danni nei rapporti di vicinanza e il pensiero dei veteres", in Studi in memoria di Emilio Albertario, I, Milano, 1953, 363, tutta la parte di testo da Dicit igitur a redigere sarebbe da attribuire all'intervento compilatorio. Diversamente si pronunciò M. SARGENTI, Actio aquae pluviae arcendae..., 56, il quale considerava genuino il riferimento al pati in quanto collegato ad una servitù di scolo a favore del fondo superiore e da esercitarsi tramite la fossa presente nel fondo inferiore.

11 Critico rispetto all'origine compilatoria del riferimento alternativo all'ipse purgare e al 
La previsione della tutela alternativa riveste un certo interesse perché permette d'individuare una sorta di contenuto minimo garantito dell'azione in questione: ove non si ritenga di poter costringere il convenuto-proprietario del fondo sul quale si trova l'opus dannoso ad intervenire personalmente su di esso, egli dovrà quantomeno permettere all'attore di provvedervi personalmente ${ }^{12}$.

Le ragioni alla base della distinzione espressa tra le due domande proponibili per mezzo dell'a. a. p. a. sono state diversamente ricostruite: si è ritenuto, da un lato, che la fossa fosse stata prevista a favore del fondo superiore, dovendo in questo caso il proprietario del fondo inferiore essere obbligato al solo patientiam praestare, anche se, ben potendo a sua volta trarre beneficio dalla presenza della fossa, avrebbe potuto provvedere direttamente alle riparazioni necessarie.

Per tale ragione, la scelta sarebbe stata lasciata al convenuto sulla base di un proprio interesse: secondo tale lettura, la condanna sarebbe stata innanzitutto ad un patientiam praestare, mentre quella al restituere suis sumptibus rappresenterebbe una possibilità sempre concessa al convenuto da valutarsi secondo le sue esigenze ed il suo interesse, il quale poteva anche essere quello di non voler permettere l'ingresso di estranei sul suo fondo, magari in ragione di rapporti non esattamente amichevoli con il proprietario del fondo vicino ${ }^{13}$.

pati in pristinum statum eam redigere si dimostra F. SITZIA, Ricerche in tema di..., 101 ss. In questo senso si veda già A. WATSON, The Law of Property in the Late Roman Republic, Oxford, 1968, 162. Si dimostrano altresì contrari F. PETERS, "Das patientiam praestare...", 152-153, L. PArenti, "Osservazioni sul "patientiam praestare"...”, 30 ss. e, rispetto al tenore generale delle sue considerazioni anche F. SCOTTI, "Actio aquae pluviae arcendae e fossae...", 300 ss.

$12 \mathrm{Nel}$ senso di essere tenuto "nur zu dem minus des patientiam praestare" si è espresso F. Peters, "Das patientiam praestare...", 152 e recentemente L. PARENTI, "Osservazioni sul "patientiam praestare"...", 33.

13 Secondo F. PETERS, "Das patientiam praestare...", 152: "Ein Interesse kann dafür ja durchaus bestehen, z.B. wenn die Nachbarn verfeindet sind und die Reinigung des

"L’obbligo di patientiam praestare nel contesto di D. 39.3.2 ..." 
Dall'altro, si è ritenuto che la decisione distinguesse tra le due forme di tutela esperibili sulla base di un differente rapporto con la fossa presente sul fondo: se questa fosse stata scavata ad esclusivo beneficio del proprietario del fondo inferiore sul quale era allocata, allora egli sarebbe stato obbligato all'ipse purgare, mentre, nel caso in cui questa fosse stata scavata nell'interesse del fondo superiore, il proprietario del fondo inferiore sarebbe stato tenuto al patientiam praestare ${ }^{14}$.

La questione dell'interesse alla presenza della fossa e del vantaggio che dalla stessa deriva per entrambi i fondi ${ }^{15}$, però, per quanto sia assolutamente necessaria per una miglior comprensione delle dinamiche di funzionamento del sistema di regolamentazione delle acque tra fondi vicini, non aiuta a cogliere del tutto, si crede, la ratio giuridica della soluzione proposta da Labeone nel caso di specie.

La quaestio giuridica qui analizzata doveva porsi sempre in termini di tutela del fondo superiore dal danno potenziale che allo stesso deriva dal fondo inferiore, in quanto la relazione tra $\mathrm{i}$ due fondi è sempre nel senso di un servire del secondo nei confronti del primo, o così, almeno, si è propensi a ritenere dalla lettura



Grabens nut mir geringer Mühe verbunden ist”. Sulla questione dell'utilità della fossa "quanto meno anche" per il fondo inferiore si esprime L. PARENTI, "Osservazioni sul "patientiam praestare"...", 33 e nota 44.

14 Questa è la tesi sostenuta da F. SITZIA, Ricerche in tema di..., 102, secondo uno schema di concessione dell'azione basato sull'utilità dell'opus già adottato per l'esegesi di D. 39.3.2.5 (Paul. 49 ad ed.) sempre in Id., Ricerche in tema di..., 95; nel caso di cui al $\int 1$, dunque, la fossa sembrerebbe non poter essere utile contemporaneamente ad entrambi i fondi.

15 Brillantemente risolta a favore dell'utilità anche per il fondo inferiore delle fossae agrorum siccandorum causa facta da F. SCOTTI, “Actio aquae pluviae arcendae e fossae...", 289 ss. e 302-303, smentendo dunque coerentemente l'impostazione del problema dell'utilità della fossa così come proposta da Peters, Parenti e Sitzia e a cui si è fatto riferimento in precedenza. 
Secondo quanto ivi indicato, la strumentalità del fondo deve essere letta in termini di $\delta o u \lambda \varepsilon i \alpha$, e non, invece, con riferimento a ciò che appare dall'osservazione dei fondi stessi $(\theta \dot{\varepsilon} \alpha)$, rilevando dunque la necessità che l'opus presente nel fondo inferiore serva all'utilità di quello superiore ${ }^{16}$. In questo senso, dunque, nel caso di specie, si concretizzerebbe quel rapporto di servizio legittimato dalla vetustas a cui fa riferimento D. 39.3.2 pr (Paul. 49 ad ed.).

La peculiarità della soluzione fornita da Labeone nel caso di specie, quindi, dovrebbe meglio ricondursi alla particolare funzione dell'opus considerato e alla prestazione alla quale il convenuto-proprietario del fondo inferiore è tenuto in relazione al rapporto che lega il suo fondo a quello superiore.

L'opus in questione, infatti, in quanto utile al fondo non può essere rimosso, e del resto, evidentemente, non avrebbe alcun senso farlo: la fossa, infatti, era stata prevista per permettere il corretto prosciugamento delle acque su entrambi i fondi affinché non si accumulassero in eccesso e allagassero o l'uno o l'altro ${ }^{17}$.

Nel caso di specie, l'interesse del proprietario del fondo superiore è dunque quello alla manutenzione della fossa, la quale, a causa del ristagno formatosi a seguito del mancato spurgo e in concorso con l'acqua piovana, rischia di danneggiare il suo fondo: non vi è dunque interesse alla rimozione della stessa, in quanto opus utile e legittimato dalla vetustas, ma lo scopo è piuttosto quello di permettere che essa riprenda il suo corretto funzionamento.

Per tale ragione, dunque, il proprietario superiore ha il

\footnotetext{
16 Si ricorda qui la distanza tra l'impostazione almeno di Giustiniano e quella classica, nella quale in tanto era esperibile l'a. $a$. $p$. $a$. in quanto i due fondi non fossero vincolati da una servitù.

17 Sul punto si veda più nello specifico F. SCOTTI, "Actio aquae pluviae arcendae e fossae...", 301-302.
}

"L'obbligo di patientiam praestare nel contesto di D. 39.3.2 ..." 
diritto di chiedere che quello del fondo inferiore, alternativamente, o provveda alla riparazione della fossa direttamente a sue spese o che gli permetta di provvedervi personalmente.

Il rilievo fondamentale è che le due soluzioni sono poste sullo stesso piano da Labeone: in questo senso ha ragione la Scotti ${ }^{18}$ quando sostiene che la correlazione aut ... aut pone sullo stesso piano le due forme alternative di restitutio, anche se il ragionamento non sembra del tutto convincente nel momento in cui l'Autrice collega il dovere del proprietario del vicino fondo inferiore a restituire suis sumptibus o a patientiam praestare al fatto che la fossa sia, correttamente, a vantaggio sia del fondo superiore sia del fondo inferiore.

Si crede qui che tale conclusione tenda a confondere i piani del discorso: non è infatti il vantaggio che il fondo inferiore riceve dalla presenza della fossa a legittimare la possibilità per il proprietario del fondo superiore di convenirlo in giudizio o per la restitutio diretta o per permettergli di procedere autonomamente alla manutenzione della fossa, ma il modo in cui si atteggia la condizione servile del fundus inferior rispetto al quello superior.

La valutazione relativa al vantaggio che la fossa può apportare anche al fondo inferiore sembra piuttosto attenere ad un momento diverso e precedente, vale a dire quello dello scavo della fossa stessa, che esso risultasse dalla lex agri ovvero che se ne sia persa memoria; per fornire un sistema di regolamentazione del deflusso delle acque più funzionale alle esigenze dei fondi, ivi compresa quella del fondo inferiore, è possibile ipotizzare che, tra le diverse soluzioni disponibili, i proprietari avessero deciso di optare per l'escavazione di un opus che permettesse al fondo inferiore stesso di ricevere le acque del fondo superiore senza

${ }^{18}$ F. SCOTTI, “Actio aquae pluviae arcendae e fossae...”, 303. 
soffrime $^{19}$; anzi, approfittando del canale per prosciugare anche quest'ultimo.

In conformità alla sostanziale parità tra i rimedi proposti, la scelta in concreto è lasciata all'attore-proprietario del fondo superiore, il quale, secondo una propria personale valutazione anche in termini di opportunità, avrebbe potuto decidere se procedere per il restituire suis sumptibus ovvero per il patientiam praestare. Ad ogni modo, il convenuto-proprietario del fondo inferiore sarebbe stato parimenti tenuto ad entrambi, in quanto la manutenzione della fossa scavata sul suo fondo era comunque dovuta per il corretto funzionamento del deflusso delle acque tra $\mathrm{i}$ due fondi, essendo il fondo di sua proprietà deputato al servizio di quello superiore.

In questo risiede, dunque, si crede qui, il senso dell'alternativa espressa per mezzo della correlazione disgiuntiva esclusiva aut ... aut. questa pone sullo stesso piano due soluzioni che hanno pari valore vincolante per il soggetto convenuto, il quale sarà tenuto all'una $\mathrm{o}$ all'altra secondo una valutazione operata in primis dall'attore-proprietario del fondo superiore.

Un discorso parzialmente diverso potrebbe condursi se l'alternativa fosse stata espressa per mezzo della correlazione digiuntiva inclusiva vel ... vel, come avviene, per esempio, nel caso considerato in

D. 39.3 .12 (Paul. 16 ad Sab.): Emptor (nisi simulata venditio est) ceterique successores vel restituere, si velint, opus factum vel patientiam praestare debent: nam actori moram suam nocere debere manifestum est. in eadem causa est etiam socius eius qui opus fecit, si ipse auctor non fuit: idemque in

${ }^{19}$ Questo conformemente alla consuetudine agricola già constatata da SICULO FLACCO, De condicione agrorum, Th. 112, 8-11 = Lach . 148, 5-8, per la quale si rimanda alle considerazioni espresse da G. FrANCIOSI, "Regime delle acque e del paesaggio...", 17.

"L’obbligo di patientiam praestare nel contesto di D. 39.3.2 ..." 
donato fundo legatove est,

e relativo all'ipotesi di un dominus nuovo acquirente del fondo obbligato - e dunque non auctor dell'opus potenzialmente dannoso - e dei suoi successori, chiamati a rispondere alternativamente tra vel restituere, si velint, opus factum vel patientiam praestare, sulla base di una valutazione personale del legittimato all'azione.

L'alternativa in questo caso si presenta in termini inclusivi, in quanto è il convenuto-acquirente del fondo - e suoi successivi aventi causa (ceteri successores) - ad avere la possibilità, in quanto del tutto estranei alle vicende che hanno causato il pericolo per il fondo vicino, di decidere sulla base del proprio interesse se optare per una restitutio diretta e personale ovvero per il semplice patientiam praestare, permettendo al vicino di rimuovere l'opus dannoso autonomamente, previo accesso al fondo.

La circostanza secondo cui, nel caso di cui al $\int 1$, il convenuto - proprietario del fondo inferiore è parimenti tenuto tanto per il restituire suis sumptibus quanto per il patientiam praestare pare ulteriormente rimarcata dalla scelta di esprimere il dovere di patientiam praestare in capo al convenuto-proprietario del fondo inferiore in temini di pati in pristinum statum eam redigere; per quanto l'argomento possa forse sembrare un po' semplicistico, il riferimento al pati sembra qui rimandare ad un dovere del proprietario del fondo inferiore che discende direttamente dalla funzione che il suo fondo assume nei confronti di quello superiore in primo luogo per la posizione e, successivamente, per l'opus che contribuisce al miglior funzionamento di tale rapporto di naturale servizio.

In questo senso, dunque, il proprietario del fondo inferiore è tenuto a sopportare che il proprietario del fondo confinante possa entrare nel suo fondo e provvedere direttamente e personalmente alla manutenzione dell'opus e al ripristino dello 
stato dei luoghi: si tratta di una forma di limitazione al suo diritto di proprietà in un senso non dissimile dall'aequo animo ferre a cui, per Labeone, il proprietario del fondo inferiore è tenuto in caso di mutamenti meramenti naturali dello stato dei luoghi, secondo quanto lo stesso giurista afferma nel noto $\int 6$ del medesimo testo paolino, in un'altra ipotesi di aquae restagnatio e conseguente necessità di procedere al suo spurgo $^{20}$ : si tratta di un dovere al quale è tenuto in virtù della relazione che lega il suo fondo a quello superiore ${ }^{21}$.

\section{Continua. D. 39.3.2.2 (Paul. 49 ad ed.): l'ipotesi della fossa scavata nei pressi della linea di confine.}

Un discorso analogo a quello appena condotto potrebbe svolgersi anche in relazione al caso di cui al successivo paragrafo:
D. 39.3.2.2 (Paul. 49 ad ed.): Praeterea si in confinio fossa sit neque purgari vicinus patiatur eam partem quae tibi accedat, posse te magis aquae pluviae arcendae Labeo ait.

Bas. 58. 13. 2. 2, VIIA

p. 2678 Scheltema: Tò






$\dot{\alpha} \nu \varepsilon \dot{\chi} \eta \tau \alpha \iota \quad x \alpha \theta \alpha \varrho \iota \sigma \theta \tilde{\eta} \nu \alpha \iota$ $\alpha \dot{\tau} \tau \dot{v}$.

\footnotetext{
${ }^{20}$ D. 39.3.2.6 (Paul. 49 ad ed.): Apud Namusam relatum est, si aqua fluens iter suum stercore obstruxerit et ex restagnatione superiori agro noceat, posse cum inferiore agi, ut sinat purgari: hanc enim actionem non tantum de operibus esse utilem manu factis, verum etiam in omnibus, quae non secundum voluntatem sint. Labeo contra namusam probat: ait enim naturam agri ipsam a se mutari posse et ideo, cum per se natura agri fuerit mutata, aequo animo unumquemque ferre debere, sive melior sive deterior eius condicio facta sit. Idcirco et si terrae motu aut tempestatis magnitudine soli causa mutata sit, neminem cogi posse, ut sinat in pristinam locum condicionem redigi. Sed nos etiam in bunc casum aequitatem admisimus.

21 Il complesso delle considerazioni sin qui svolte porta a non accogliere la ricostruzione della soluzione labeoniana così come proposta da Peters e adesivamente seguita da Parenti, precedentemente ricordata, seppur, come si è visto, in termini parzialmente diversi da quelli espressi da F. SCOTTI, "Actio aquae pluviae arcendae e fossae...", 302-303. In questo caso non esiste una scala di valori tra le due soluzioni :queste sono poste sullo stesso piano da Labeone, ed esse tutelano la medesima fattispecie, cioè la manutenzione della fossa, poiché trattandosi di fossa agrorum siccandorum causa è stata comunque scavata nel'interesse di entrambi.
}

"L’obbligo di patientiam praestare nel contesto di D. 39.3.2 ..." 
Il caso di specie si differenzia da quello di cui al $\int 1$ per il fatto che la fossa si trovava praticamente sulla linea di confine tra i due fondi: il vicino frontista si rifiutava di pati, di sopportare che il proprietario del fondo superiore provvedesse allo spurgo della fossa dalla parte che si affacciava sul suo lato; in questo senso pare debba intendersi eam partem quae tibi accedat di cui al testo.

In entrambi i casi considerati da Paolo si ritrovano il naturale rapporto di servizio tra $i$ due fondi dato dalla posizione di superiorità dell'uno rispetto all'altro, l'opus vetus funzionale al prosciugamento dell'acqua in eccesso e la sua posizione nel fondo inferiore, per quanto nei pressi, appunto, della linea di confine ${ }^{22}$.

In questa ipotesi, Labeone avrebbe suggerito che il rimedio esperibile per il proprietario del fondo inferiore avrebbe dovuto essere $1^{\prime} a$. a. p. a.: tale rimedio nel testo di Paolo è introdotto dal termine magis, il cui utilizzo implica una comparazione rispetto alla quale tale azione avrebbe dovuto essere preferita, ipoteticamente, ad un altra, di cui manca però il riferimento.

Tale lacuna ha originato in dottrina una serie cospicua di proposte integrative; tra queste, Fokke Hinrichs ${ }^{23}$ suggerisce che la soluzione proposta da Labeone posse te magis aquae pluviae arcendae Labeo ait debba essere riscostruita nel senso posse te magis

\footnotetext{
22 Sul punto si veda da ultimo F. SCOTTI, “Actio aquae pluviae arcendae e fossae...”, 281 ss. e 304 ss., la quale ritiene che la fossa non si trovi sul confine, ma nei pressi, rilevando coerentemente come nel primo caso si sarebbe trattato di una fossa in comune, già di proprietà per la sua parte del dominus del fondo superiore che avrebbe potuto provvedere dal suo lato allo spurgo della fossa.

$23 \mathrm{Si}$ veda F. T. HinRICHS, Die Geschichte der gromatischen Institutionen: Untersuchungen zu Landverteilung, Landvermessung, Bodenverwaltung und Bodenrecht im römischen Reich, Wiesbaden, 1974, 178 e nota 37, anche nella traduzione francese F. T. HiNRICHS, Histoire des institutions gromatiques, Édition assurée par le Centre d'Histoire Ancienne de Besançon. Traduction D. Minary, texte revu par Gérard Chouquer, Monique ClavelLévêque et François Favory avec la collaboration de Marcel Morabito, Paris, 1989, 188 e nota 37.
} 
$<$ actione $>$ aquae pluviae arcendae $<$ quam ille finium regundorum $>$ Labeo ait.

\section{Il magis introdurrebbe dunque una preferenza per l'esercizio} preventivo dell'a. a. p. a. da parte del proprietario del fondo superiore rispetto, invece, alla possibilità che il dominus del fondo inferiore eserciti contro di lui l'actio finium regundorum: l'Autore ipotizza infatti che, data la vicinanza della fossa al fondo, la quaestio giuridica fosse collegata ad un'opposizione da parte del proprietario del fondo inferiore alle attività di spurgo sulla linea di confine compiute dal dominus del fondo superiore allo scopo di evitare che questi usucapisse il confinium stesso. Per fare ciò avrebbe quindi potuto esercitare l'actio finium regundorum, impedendo al vicino di riparare la fossa e, conseguentemente, di usucapire il confine ${ }^{24}$.

24 Ulteriori e diverse ricostruzioni sono state in precedenza proposte in dottrina; secondo T. Mommsen, P. Krueger, Digesta Iustiniani Augusti..., II, 397, l'opinione di Labeone andrebbe emendata nel senso di posse te magis $<$ in rem agere quam $>$ aquae pluviae arcendae Labeo ait, proponendo l'esercizio di un'actio in rem quale l'actio negatoria piuttosto che l'actio aquae pluviae arcendae per permettere al proprietario del fondo superiore di porre fine alla turbativa all'esercizio del suo diritto di proprietà causata dall'opposizione del proprietario del fondo inferiore allo spurgo della fossa. Secondo F. SCOTTI, "Actio aquae pluviae arcendae e fossae...", 280, tale peculiare ricostruzione deriverebbe dal considerare la fossa in questione come scavata sul fondo superiore e non su quello inferiore. Secondo O. LENEL, Palingenesia Iuris Civilis, iuris consultorum reliquiae quae Iustiniani digestis continentur ceteraque iuris prudentiae civilis fragmenta minora secundum auctores et libros, I, Lipsia, 1889, 1058 e nota 2, il testo dovrebbe invece essere letto nel senso di posse te magis <agere finium regundorum quam> aquae pluviae arcendae Labeo ait, sul presupposto che la fossa si trovasse sul fondo superiore, seppur attaccata al confine, ragion per cui la maggior convenienza nell'agire con l'actio finium regundorum stava nella possibilità per il proprietario del fondo superiore di far dichiarare la fossa come finalis e di sua proprietà, come sottolinea sempre F. SCOTTI, "Actio aquae pluviae arcendae e fossae...", 281. Secondo la ricostruzione proposta da M. SARGENTI, Actio aquae pluviae arcendae..., 56, infine, il testo dovrebbe essere letto nel senso di posse te magis $<$ in rem agere $>$ [aquae pluviae arcendae] Labeo ait, conformemente alla sua ricostruzione del caso di cui al $\int 1$ come una servitù di scolo dell'acqua, che avrebbe dunque legittimato all'esercizio di un'actio in rem: essa sarebbe stata un'actio confessoria nell'ipotesi in cui la fossa fosse stata scavata interamente nel fondo inferiore, al fine di permettere al vicino l'accesso al fondo e l'esecuzione delle opere di spurgo, ovvero un'actio negatoria nell'ipotesi in cui la fossa si fosse trovata in parte nel fondo superiore e il dominus del

"L’obbligo di patientiam praestare nel contesto di D. 39.3.2 ..." 
Labeone, invece, sostiene che il proprietario del fondo superiore abbia il diritto di esercitare nei confronti del vicino l'a. a. p. a. negli stessi termini di cui al $\$ 1$ (il praeterea si con cui si apre il \2), vale a dire l'agire per il restituere suis sumptibus ovvero il pati in pristinum statum eam redigere, che il proprietario del fondo inferiore si rifiuta di prestare: egli ha alternativamente diritto a domandare entrambe le forme di tutela, con il corrispondente obbligo per il convenuto di prestarle, in virtù della funzione assegnata alla fossa legittimata dalla vetustas nell'economia dei rapporti tra $\mathrm{i}$ due fondi e del dovere in capo al dominus del fondo inferiore di mantenerla in buono stato per il regolare svolgimento delle attività sui fondi, non essendo d'impedimento a ciò la peculiare posizione di confine della fossa stessa.

\section{La ricostruzione del $\int 2$ così come proposta da Hinrichs}

vicino fondo inferiore avesse impedito lo spurgo proprio di questa parte. Secondo quanto rilevato da F. SITZIA, Ricerche in tema di..., 102 ss. e nota 103, adesivamente seguito da L. PARENTI, "Osservazioni sul "patientiam praestare"...", 36 e nota 46, e da ultimo da F. SCOTTI, "Actio aquae pluviae arcendae e fossae...", 306, la ricostruzione proposta da Hinrichs troverebbe conferma nel corrispondente testo dei Basilici di cui a Bas. 58.13.2.1-2 di cui al testo. In verità sembrerebbe che il testo complessivo che si legge nei Basilici, come già evidenziato, confermi l'impostazione generale dei due testi come sopra descritta, da cui la ricostruzione dell'ipotesi di cui al $\ 2$ nei termini proposti da Hinrichs trae origine, ma non spiega l'individuazione della ratio giuridica alla base della sua ricostruzione. Individuata coerentemente la fossa sul fondo inferiore, ed essendo estranea all'ipotesi in oggetto la struttura della servitù, il secondo termine di paragone introdotto da Labeone doveva necessariamente ricondursi ad un problema di confini, e dunque conseguentemente all'actio finum regundorum. Per come lo leggiamo noi, il testo è stato sicuramente rimaneggiato in un momento successivo, togliendo dall'opinione di Labeone il riferimento al secondo termine di paragone, l'actio finum regundorum, in quanto probabilmente non più attuale; come sottolinea l'Autore, è infatti una costituzione del 385 d.C., CTh. 2.26.4, che sancisce l'interdizione dell'usucapio del confinium per gli agri arcifinii. Una volta venuto meno tale limite all'usucapione della zona di confine nel limite di 5 piedi, la questione non doveva più presentarsi nei termini esposti (una difesa del proprietario del fondo inferiore dal tentativo del dominus del fondo superiore di usucapire il confine per mezzo dell'attività di spurgo) e si ritenne probabilmente di espungere il secondo termine di paragone, lasciando che il casus trovasse inquadramento solo in termini di esercizio dell'a. a. p. a., anche conformemente al nuovo ruolo di generale regolamentazione dei rapporti tra fondi assunto dall'actio nel regime giustinianeo. 
pare di fatto unanimemente accolta in dottrina ${ }^{25}$; sarebbe però veramente del tutto scorretto leggere il responso di Labeone come se l'espunta actio finum regundorum fosse riferita al proprietario del fondo superiore, e venisse dunque a mancare il riferimento all'ille così come integrato da Hinrichs?

Se il caso del $\int 2$, come già più volte sottolineato, è lo stesso di cui al $\int 1$ per quanto attiene ai presupposti di fatto, la questione giuridica centrale resta sempre quella della mancata manutenzione di un opus vetus della cui costituzione non si ha memoria, non previsto dalla lex agri ma legittimato dalla vetustas, che deve essere reso nuovamente funzionale per permettere che $\mathrm{i}$ fondi siano prosciugati correttamente: l'unica differenza si riscontra nella posizione della fossa, che invece di trovarsi all'interno del fondo inferiore in un punto imprecisato, si trova attaccata alla linea di confine.

Differentemente dal caso di cui al $\int 1$, in virtù del quale, senza l'equiparazione della forza cogente della vetustas a quella della lex agri che legittimasse l'opus, il proprietario del fondo inferiore non avrebbe avuto il diritto di esperire alcuna azione contro il proprietario del fondo inferiore per ottenere che questi spurgasse la fossa o gli permettesse di farlo, in quanto opus manu factum non previsto dalla lex agri, nel caso del $\int 2$ il fatto che la fossa fosse posta in prossimità del confine comportava che al proprietario del fondo superiore, a fronte del rifiuto a sopportare (pati) lo spurgo della fossa opposto dal proprietario del fondo

\footnotetext{
25 Anche L. PARENTI, “Osservazioni sul “patientiam praestare”...”, 36 e nota 46, come si è già rilevato nella nota precedente, aderisce alla ricostruzione del responso di Labeone nei termini proposti da Hinrichs; però, come rileva anche F. SCOTTI, "Actio aquae pluviae arcendae e fossae...", 282 e nota 40, egli legge il testo nel senso che fosse il proprietario del fondo superiore che si assumeva potenzialmente danneggiato dalla mancata manutenzione della fossa vetus a dover preferire l'esercizio dell'a. a. p. a. a quello dell'actio finum regundorum, omettendo il riferimento all'ille emendato da Hinrichs. Si tratta, molto probabilmente, di un'inconguenza frutto di una semplice svista, anche perché tale mutamento nell'esegesi del testo non pare supportato da alcuna argomentazione.
}

"L'obbligo di patientiam praestare nel contesto di D. 39.3.2 ..." 
inferiore per evitare l'usucapione del confinium, sarebbe sempre rimasta la possibilità di coinvolgere il vicino in una disputa sui confini ed agire con l'actio finium regundorum per chiedere che il confine venisse rideterminato (in suo favore), trattandosi di agri arcifinii e dunque, privi come erano di limites, confinanti direttamente tra loro.

In questo modo, una volta tracciata la nuova linea di confine in suo favore, con l'effetto di trasformare la fossa di confine in una fossa in comproprietà, egli avrebbe avuto diritto di procedere alle attività di manutenzone senza che il vicino potesse opporre alcunché, non trattandosi più di un'attività svolta su fondo altrui ma su fondo proprio.

Ammettendo la possibilità di esperire l'a. a.p. a. nei termini di cui al $\int 1$ anche nel caso di cui al $\int 2$, Labeone (e con egli Paolo), forniva una tutela più consona al proprietario del fondo che si assumeva potenzialmente danneggiato dal mancato spurgo della fossa, basata sul rapporto di vicinanza dei fondi, costringendo il vicino a quel pati al quale si opponeva, e permettendogli dunque d'intervenire direttamente per ottenere la manutenzione dell'opus senza dover agire per l'adiudicatio. Nulla, però, impediva al dominus di agire per la modificazione dell'assetto del confine in suo favore, qualora ne avesse avuto l'interesse, ovvero il vicino ritenesse di doversi opporre ad un tentativo di usucapione del confinium, ragion per cui, ancora in età classica, il riferimento all'actio finium regundorum dovette rimanere espressamente previsto.

Probabilmente, solo in in sede compilatoria e alla luce del progressivo disuso dell'actio in questione, dell'intervenuta interdizione dell'usucapio del confinium e del parallelo configurarsi dell'a. a. p. a. quale strumento generale di regolamentazione dei rapporti tra vicini, si dovette ritenere il riferimento all'azione di regolamentazione dei confini desueto e ormai privo di rilevanza 
giuridica, al punto da espungerlo dal testo. Il primo termine di paragone, del resto, è introdotto da magis e non da prior, che parrebbe invece il senso attribuitogli da Hinrichs.

\section{Considerazioni conclusive.}

I testi qui analizzati ci permettono ora di trarre alcune conclusioni in ordine alle condizioni di esercizio dell'esercizio dell'a a. $p$. a. ed al loro rapporto con una miglior gestione del fondo rurale.

Si è visto come sino dai primi due paragrafi del testo di cui a D. 39.3.2 si affrontino diverse questioni di peculiare rilievo per la determinazione delle condizioni di esercizio dell'a. a. p. a.: rispetto al principio generale che apre il testo di Paolo secondo cui, come si è visto, il fondo inferiore serve il fondo superiore secondo quanto stabilito dalla lex, dalla natura loci e dalla vetustas, il prosieguo del ragionamento si sviluppa attorno ad alcune peculiari e controverse applicazioni pratiche dello stesso, rispetto alle quali rilevano altresì profili più strettamente collegati al titolo di responsabilità imputabile al convenuto.

La condanna di quest'ultimo, si è visto, si può atteggiare in modi differenti, prevedendo che fosse egli stesso a restituere suis sumptibus ovvero, in taluni casi e in particolari circostanze, a permettere che fosse l'attore a provvedere da sé al ripristino della situazione nello stato precedente al suo mutamento ${ }^{26}$.

Nei termini in cui la questione si presenta nell'ambito del testo di Paolo si può rilevare come, in alcune ipotesi, l'a. $a$. $p$. $a$. comportasse una sorta di quantum minimo di tutela riconducibile al dovere generale di patientiam praestare e che dunque l'esercizio

\footnotetext{
26 Già E. SCHÖNBAUER, "Die actio aquae pluviae arcendae. Ein Beitrag zur Geschichte des römischen Agrarrechtes", ZSS 54, 1934, 254 ss., aveva distinto tra i diversi modi in cui il iussum indicis poteva esprimersi: nei casi qui analizzati pati, fossam purgare, aggerem reponere, aggerem reponi sinere, agrum purgare, purgari sinere, fossam reficere.
}

"L’obbligo di patientiam praestare nel contesto di D. 39.3.2 ..." 
dell'azione, ove ritenuta esperibile, fosse volta a garantire al convenuto almeno il diritto di provvedere da sé alla restitutio.

Diversamente dai casi in cui l'obbligo in questione viene definito espressamente in termini di patientiam praestare in corrispondenza di alcune ipotesi specifiche dove assumono rilievo le peculiari vicende relative alla proprietà del fondo obbligato (colonia, comproprietà, trasferimenti di proprietà) e l'obbligo in questione assume i contorni più generali di un dovere minimo in capo al proprietario del fondo obbligato, nei casi che concorrono alla costruzione di D. 39.3.2 (Paul. 49 ad ed.) il discorso si fa più complesso, in quanto la possibilità per il proprietario che si assume danneggiato di provvedere da sé al ripristino dello stato dei luoghi assume rilievo in modo differente a seconda del caso considerato.

Essa è oggetto di una specifica previsione che presuppone a monte una valutazione della posizione reciproca dei fondi; del tipo di opus che viene in rilievo; del tipo di intervento (manutenzione o distruzione) che conseguentemente è oggetto della richiesta in via d'azione.

Nelle due peculiari ipotesi di aquae restagnatio analizzate, la possibilità di esperire l'a. a. p. a. onde evitare i danni del mancato spurgo da parte del proprietario del fondo delle fossa adibita al prosciugamento dei campi collocata sulla sua proprietà è determinata da un intevento di tipo manutentivo.

La fossa, infatti, sia che essa si trovi completamente su uno dei fondi confinanti come nell'ipotesi di cui al $\int 1$ (e nello specifico sul fondo inferiore) sia che si trovi invece in prossimità della linea di confine come nell'ipotesi di cui al $\$ 2$, l'opus artificiale era utile alla corretta gestione dei fondi coinvolti.

Per tale ragione, evidentemente, l'actio in questione non 
avrebbe dovuto essere esperita per la demolizione dell'opus potenzialmente dannoso, in quanto tale circostanza avrebbe irrimediabilmente mutato lo stato dei luoghi ed il rapporto di funzionalità reciproca tra i fondi, in violazione anche del precetto di cui al principium del testo paolino.

In entrambi i casi l'azione era dunque concessa per preservare la fossa ed il suo corretto utilizzo, legittimando il proprietario del fondo potenzialmente danneggiato a chiamre in giudizio il proprietario del fondo confinante affinch aut ipse purgaret aut te pateretur in pristinum statum eam redigere, prevedendo un'alternativa tra il patientiam praestare e la diretta rimessione in pristino dello stato della fossa da parte del proprietario del fondo su cui la fossa si trovava allocata.

Tale obbligo al patientiam praestare, differentemente dagli altri casi in cui esso era previsto nell'ambito del titolo III del libro 39 del Digesto, non è inquadrato come una sorta di tutela sussidiaria per il proprietario del fondo confinante potenzialmente danneggiato dall'aqua pluvia in caso di mutamento nella titolarità del fondo obbligato: il domino non auctor, infatti, non avrebbe potuto procedere alla restitutio sui sumptibus.

$\mathrm{Nel}$ caso in cui al $\ 1$, invece, il patientiam praestare assume piuttosto i contorni di un contenuto minimo dell'a. a.p.a. a., il cui effettivo esperimento è rimesso alla volontà del proprietarioattore.

Come ben evidenziato dalla correlazione disgiuntiva esclusiva aut ... aut, le due soluzioni sono messe sullo stesso piano e restano dunque alternative (seppur in senso esclusivo) per l'attore, il quale può decidere quale di questo sia maggiormente conforme al proprio interesse ed al tempo stesso parimenti vincolanti per il proprietario del fondo (inferiore) convenuto, in quanto la manutenzione della fossa scavata sul suo fondo era 
comunque dovuta per il corretto funzionamento del deflusso delle acque tra $\mathrm{i}$ due fondi, essendo il fondo di sua proprietà deputato al servizio di quello superiore.

Per tale ragione egli può essere obbligato a pati, a sopportare comunque l'intervento invasivo e cautelativo del confinante quale forma di limitazione del suo diritto di proprietà per la migliore gestione del fondo.

Stesso ragionamento può essere condotto con riferimento al $\int$ 2; la possibilità di esercitare l'a. a.p. a. anche con rifermento al pati, in capo al vicino, dello spurgamento diretto della fossa, in luogo dell'ormai desueto intervento con l'actio finium regundorum, ricompone l'alternativa tra le due forme di tutela esperibili, che anche in questo caso, essendo volte alla manutenzone di un opus utile, hanni pari valore e sono rimesse alla valutazione del proprietario-attore.

Con la presente breve analisi si è dunque cercato di dimostrare come il contenuto dell'a. a. p. a., in ipotesi peculiari di opus artificiale potezialmente dannoso ma la cui presenza è necessaria per la funzionalità del fondo,poteva essere concessa per la sua manutenzione, arricchendosi nel suo contenuto.

L'obbligo, infatti, a prescindere da ogni vicenda legata alla titolarità del fondo, poteva essere esteso, a discrezione del proprietario-attore, ad un dovere genericamente identificato come patientiam praestare, ma che di fatto consisteva in un pati, in un sopportare che da un lato limitava il diritto di proprietà del proprietario-convenuto, ma dall'altro era volta alla corretta e fruttuosa gestione del fondo, nel rispetto del rapporto di funzionalità che legava $i$ fondi confinanti. 


\section{Bibliografia}

A. Berger, v. Patientiam praestare, in Encyclopedic Dictionary of Roman Law, Philadelphia 1953 (rist. 1991), 620 e ripresa da L. PARENTI, "Osservazioni sul "patientiam praestare" in Labeone", in Teoria e storia del diritto privato (http:/ / www.teoriaestoriadeldirittoprivato.com), V (2012), 1

P. BONFANTE, Corso di diritto romano. La proprietà, vol. II, Milano 1966 (ristampa corretta della I edizione a cura di Giuliano Bonfante e di Giuliano Crifò con l'aggiunta degli indici delle fonti)

R. CARDILLI, L'obbligarione di «praestare» e la responsabilità contrattuale in diritto romano (II sec. a.C. - II sec. d.C.), Milano, 1995

A. Di PORTO, "La tutela della "salubritas" fra editto e giurisprudenza. Il ruolo di Labeone. I - Acque", BIDR 91, 1988, 459-570

A. Di PORTO, "La tutela della "salubritas" fra editto e giurisprudenza. Il ruolo di Labeone. II. Cloache e salubrità dell'aria”, BIDR 92, 1989, 271-309

A. DI PORTO, La tutela della "salubritas" fra editto e giurisprudenza: Il ruolo di Labeone, Milano, 1990

M. FIORENTINI, "Precedenti di diritto ambientale a Roma? I. la contaminazione delle acque", Index 34, 2006, 353-400

M. FIORENTINI, "Equilibri e variazioni ambientali nella prospettiva della tutela processuale romana", in E. HERMON (dir.), Société et climats dans l'Empire romain. Pour une perspective historique et systémique de la gestion des ressources en eau dans l'Empire romain, Préface de L. LABRUNA, Napoli, 2009, 69-111

G. FRANCIOSI, "Regime delle acque e del paesaggio in età repubblicana", in Uomo, acqua e paesaggio. Atti dell'incontro di studio sul tema "Irregimentarione delle acque e trasformazione del paesaggio antico". S. Maria Capua Vetere - 22-23 novembre 1996, Roma, 1997, 11-19

F. T. HINRICHS, Die Geschichte der gromatischen Institutionen: Untersuchungen zu Landverteilung, Landvermessung, Bodenverwaltung und Bodenrecht im römischen Reich, Wiesbaden, 1974

F. T. HINRICHS, Histoire des institutions gromatiques, Édition assurée par le Centre d'Histoire Ancienne de Besançon. Traduction D. Minary, texte revu par Gérard Chouquer, Monique Clavel-Lévêque et François Favory avec la collaboration de Marcel Morabito, Paris, 1989

O. LENEL, Palingenesia Iuris Civilis, iuris consultorum reliquiae quae Iustiniani digestis continentur ceteraque iuris prudentiae civilis fragmenta minora secundum auctores et libros, I, Lipsia, 1889

F. MusumeCI, "Tutela dell'ambiente e diritto romano", Index 20, 1992, 564-574

"L’obbligo di patientiam praestare nel contesto di D. 39.3.2 ..." 
F. PETERS, "Das "patientiam praestare" im klassischen römischen Nachbarrecht", SDHI 35, 1969, 135-214

M. SARGENTI, Actio aquae pluviae arcendae. Contributo alla dottrina della responsabilità per danno nel diritto romano, Milano, 1940

E. SCHÖNBAUER, "Die actio aquae pluviae arcendae. Ein Beitrag zur Geschichte des römischen Agrarrechtes", ZSS 54, 1934, 233-257

F. SITZIA, Ricerche in tema di "actio aquae pluviae arcendae". Dalle XII tavole all'epoca classica, Milano, 1977

F. SCOTTI, "Diritto e agronomi latini: un caso in tema di actio aquae pluviae arcendae", Agri Centuriati 10, 2013, 9-39

F. SCOTTI, "Actio aquae plwiae arcendae e fossae agrorum siccandorum causa factae: per un'esegesi di D. 39.3.2.1,2,4,7 alla luce delle tecniche agronomiche antiche", Jus. Rivista di Scienze Giuridiche 2, 2014, 273-308

F. SCOTTI, "Brevi riflessioni su Dig. 39.3.1.4,5,9 Ulp. 53 ad ed.", RIPARLA 1, 2015, 133-159

F. SCOTTI, "Actio aquae plwiae arcendae e «piccola bonifica agraria»: un esempio dalle fonti giustinianee", in Mater Familias. Scritti per Maria Zabtocka, Warszawa, 2016, 725 ss. = JusOnline 3, 2016, 752-783

A. WATSON, The Law of Property in the Late Roman Republic, Oxford, 1968 W. WOLODKIEWICZ, "Protection de l'environnement naturel et gestion de l'eau d'hier et d'aujourd'hui', in R. BEDON, E. HERMON (éds.), Concepts, pratiques et enjeux environnementaux dans l'Empire romain, Limoges, 2005, 53-66 